University of St Andrews
STACEES

St Andrews Network for Climate, Energy, Environment and Sustainability

The Sustainability Series

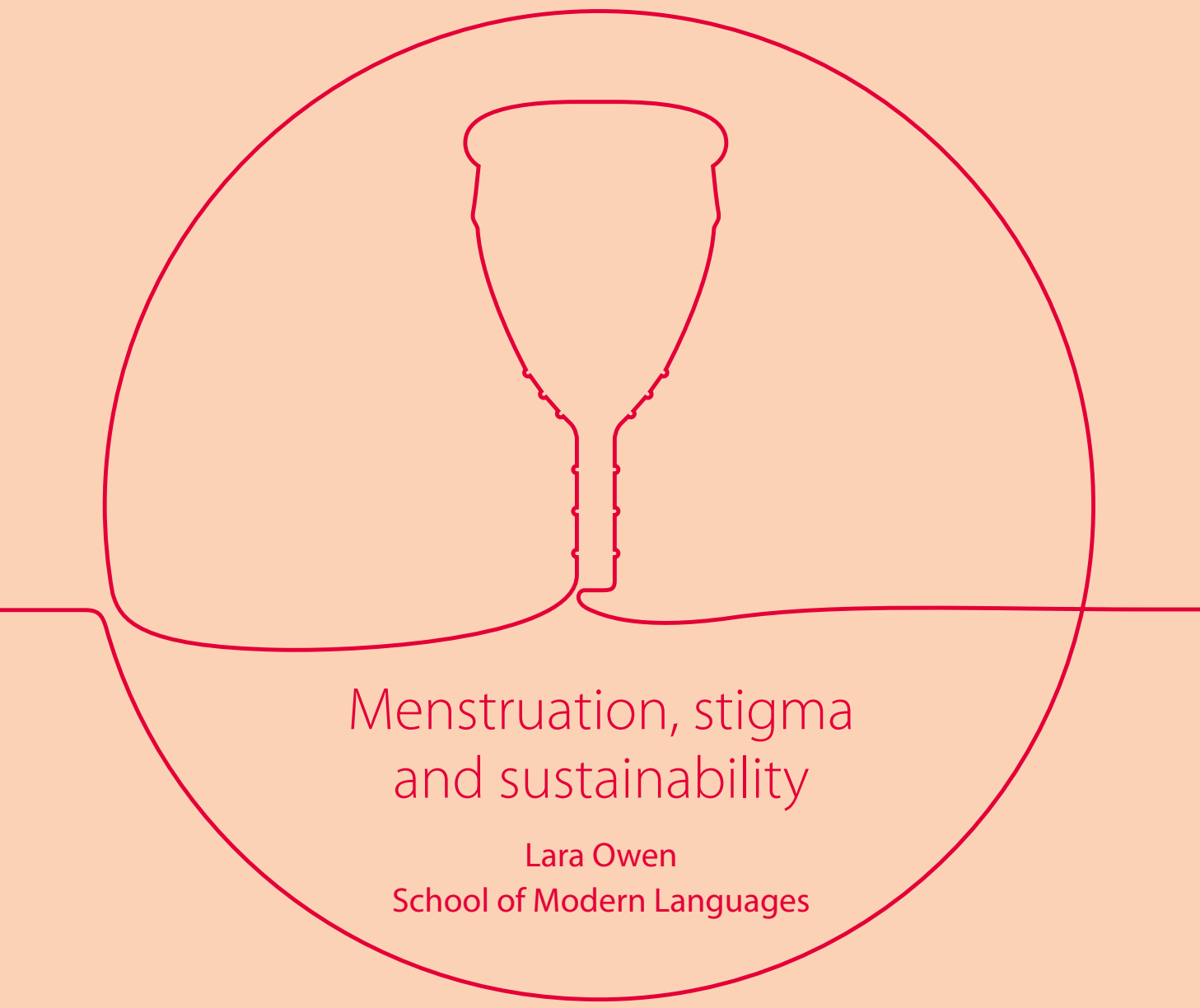




\title{
Menstruation, stigma and sustainability
}

\author{
Lara Owen - School of Modern Languages \\ (Article written by Garry MacKenzie) \\ Copyright @ University of St Andrews 2021. For full terms, see end of article.
}

Finding ways to reduce the human impact on the environment means looking at every aspect of our lives and asking what can be done to make them more sustainable. Sometimes, this task needs to address everyday activities that are not usually discussed. Menstruation is one such under-researched topic; historically stigmatised, it has been given little academic or wider public attention. This has resulted in a lack of knowledge about how women, trans and non-binary people manage their menstrual cycles - whether in terms of choosing which kinds of menstrual products to use and the subsequent disposal of these products, or how employment practices can both help and hinder those who menstruate.

Research at the University of St Andrews is seeking to address this lack of knowledge. At the School of Modern Languages, Dr Lara Owen and Professor Bettina Bildhauer are investigating how culture affects the use and disposal of period products. Funded by the St Andrews Restarting Research Funding Scheme, their project is titled 'Menstrual cups vs tampons: an interdisciplinary analysis of barriers and changing attitudes to sustainable period products in contemporary Scotland'. It asks whether the ongoing stigmatisation of menstruation is a major factor that deters some menstruators from disposing of period products sustainably. It also asks whether stigmatisation stops more menstruators from choosing reusable products (including menstrual cups). Owen's academic background is in the field of organisation studies and this project is part of her ongoing research into innovations in the organisation of menstruation.

\section{Plastics and the marine environment}

Access to menstrual products or the choice of which products to use may seem like social or economic issues rather than issues related to environmental sustainability. However, recent research from the Scottish Government shows that disposable period products (tampons and single-use pads) are a major source of marine pollution in Scotland. In a public survey, around one quarter of the respondents told researchers that they either always or frequently flush tampons down the toilet. A significant number also flush sanitary towels. These products can not only block the sewage network, but also find their way into rivers and oceans. Many tampons and pads contain plastics that contaminate marine ecosystems, and they are often disposed of in ways that maximise concealment and minimise environmental protection.

There is rising interest in sustainable alternatives, such as menstrual cups. 
Still, reusable period products remain less popular than disposable ones. The reasons for this include: disposables brands dominating retail and advertising space; greater initial cost (although reusable products are cheaper in the long run); uncertainty about switching from a familiar product; and issues relating to poverty and menstrual stigma, such as lack of access to private bathroom facilities.

\section{Language, stigma and cultural change}

Language plays an important role in shaping and reflecting our attitudes and identities. So, linguistic analysis of the stigmatisation of menstruation is a vital tool for better understanding and normalising the topic of menstruation. The 'Menstrual cups vs tampons' project is an interdisciplinary study involving sociological and linguistic analyses of menstrual practices in contemporary Scotland. It includes interviews with representatives from the environmental organisations Zero Waste Scotland and Resource Futures, as well as an analysis of the language relating to menstruation used online by Scottish period product manufacturers.

The project also seeks to understand what consultants, campaigners and manufacturers have to say about issues related to sustainability and the stigmatisation of menstruation. What are their concerns and goals in these areas, and what lessons have they taken on board through their own experience and research? How aware are they of menstrual stigma as a factor in the selection of period products?

Delving into questions of stigma and sustainability, the project investigates the growing use of menstrual cups and reflects on ongoing barriers to greater uptake of this sustainable period product. Owen's previous fieldwork in Australia found that sustainability was a selling point, making menstrual cups acceptable and even 'cool' to young users and their peer groups.

Owen's research further explores workplace management. When the menstrual cycle and menopause are not factored into working conditions (as reasons for leave or flexible working, for example), this can impact both employee wellbeing and staff turnover. As with the sustainability of period products, this topic is relatively understudied and underdiscussed. In response, Owen's research explores how menstrual stigma contributes to the silence around menstruation at work and how this can be overcome.

\section{St Andrews, Scotland and beyond}

This research into issues of stigma and sustainability is part of the growing academic field of Critical Menstrual Studies, which seeks to address the relative neglect of the topic as a subject of scholarly study. With its Menstruation Research Network, the University of St Andrews is establishing itself as a leading international centre of expertise in the field. Within the study of menstruation, the network is distinctive 
in its embrace of the humanities as well as the social and medical sciences.

Globally, the Scottish Government is at the forefront of destigmatising menstruation in public life. In landmark legislation aimed at ending period poverty, Scotland has become the first country in the world to enshrine in law a universal right of access to free period products. Local authorities, schools, colleges and universities are legally committed to ensuring that tampons and pads are available to anyone who needs them.
The research from Owen and Bildhauer will contribute to the process of destigmatisation within Scotland and beyond, educating people about reusable period products and specifying how work and learning environments can become more menstruationfriendly. As people around the world reflect on ways to reduce their impact on the environment, the project sheds light on how this question might apply to one of the most intimate and underdiscussed aspects of everyday life.

\section{Find out more}

Researcher website: $\underline{\text { http://laraowen.com }}$

Researcher Twitter: https://twitter.com/laraowen

Researcher Linkedln: www.linkedin.com/in/laraowen/

Research Network Twitter: https://twitter.com/menstruationRN 
Publisher: St Andrews Network for Climate, Energy, Environment and Sustainability, University of St Andrews.

\section{Editors: Sarah Bennison and Laura Pels Ferra.}

Copyright: $\odot$ University of St Andrews 2021. All rights reserved. Licences issued by the Copyright Licensing Agency Limited do not extend to this work. The reproduction or transmission of all or part of the article, whether by photocopying or storing in any medium by electronic means or otherwise, without the written permission of the owner, is prohibited. The commission of any unauthorised act in relation to the work may result in civil or criminal actions.

Disclaimer: This article is one of a series of articles collectively entitled 'The Sustainability Series', written for STACEES, the St Andrews Network for Climate, Energy, Environment and Sustainability. STACEES is a research network at the University of St Andrews. The articles contained in the Series are based on research and may also contain opinion-based information. The opinions stated herein do not necessarily reflect the ideas, ideologies, or points of view of the Editors, the University of St Andrews or any other organisation.

\section{Neither the University of St Andrews nor the Editors or Author(s):}

- makes any warranties about the completeness, reliability and accuracy of the information contained herein and in particular takes no responsibility for the accuracy or otherwise of any part of the information contained herein (including any claims made herein) or for any errors or missing information. Any reader who relies on the information or views expressed in the article does so at their own risk. Any action taken as a result of the information or views contained in the article is at the reader's risk;

- will be liable for any losses and/or damages whether directly or indirectly from or arising in connection with the use of this article; and

- will be held responsible for any misuse, reuse, recycled and cited/uncited copies of content from by others.

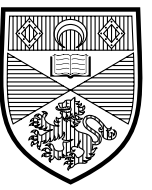

University of St Andrews
The St Andrews Network for Climate, Energy, Environment and Sustainability (STACEES) is an interdisciplinary research-focused initiative launched in April 2021 at The University of St Andrews.

The Sustainability Series comprises 15 professionally written, accessible articles commissioned by STACEES to showcase the breadth of the world-leading sustainability-focused research at the University.

Designed and produced by University of St Andrews Print \& Design Unit, September 2021. The University of St Andrews is a charity registered in Scotland. No: SC013532. 